This item was submitted to Loughborough's Institutional Repository (https://dspace.lboro.ac.uk/) by the author and is made available under the following Creative Commons Licence conditions.

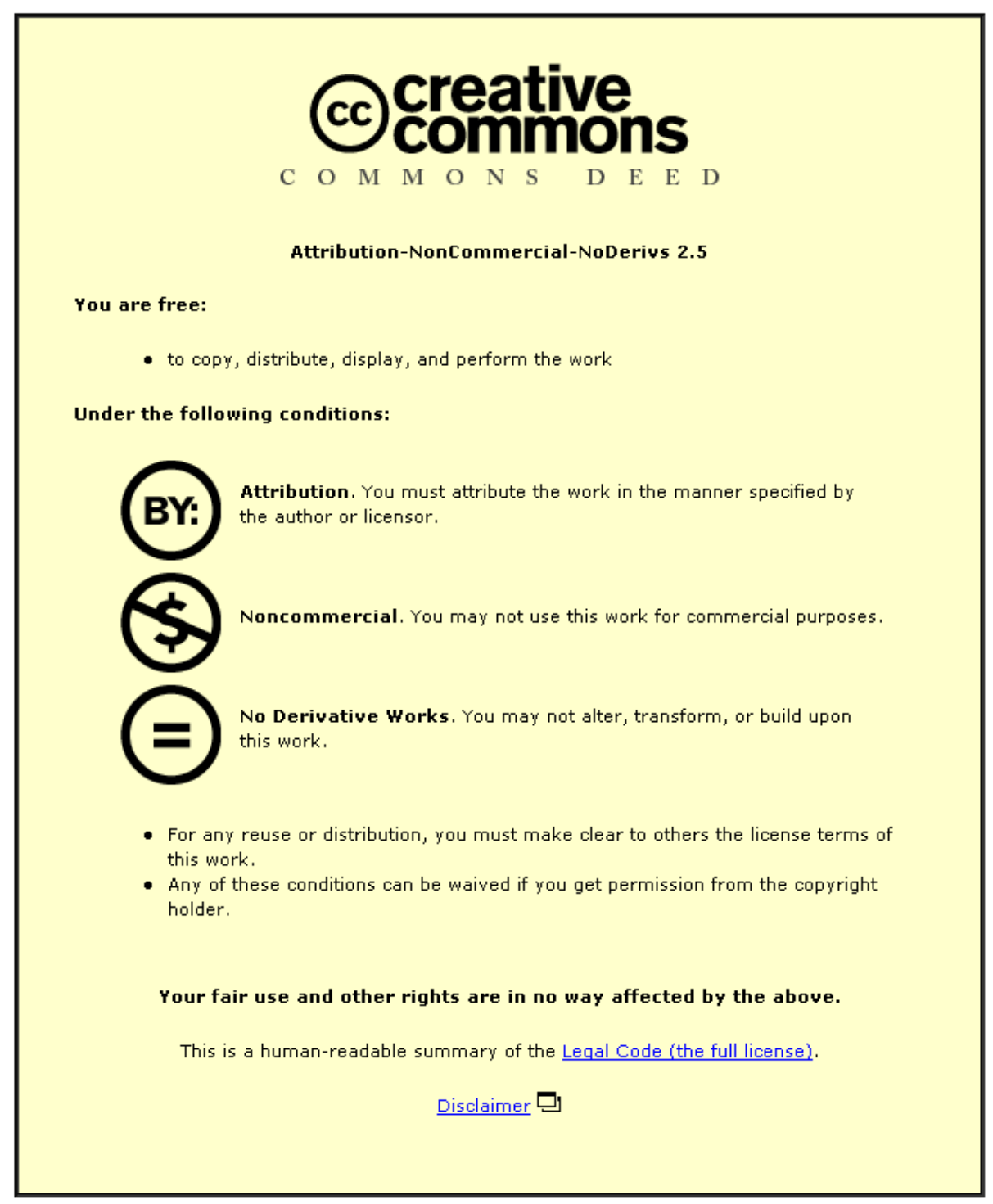

For the full text of this licence, please go to: http://creativecommons.org/licenses/by-nc-nd/2.5/ 


\title{
Polaronic memory resistors strongly coupled to electrodes
}

\author{
A. S. Alexandrov ${ }^{1}$ and A. M. Bratkovsky ${ }^{2}$ \\ ${ }^{1}$ Department of Physics, Loughborough University, Loughborough LE11 3TU, United Kingdom \\ ${ }^{2}$ Hewlett-Packard Laboratories, 1501 Page Mill Road, Palo Alto, California 94304, USA
}

(Received 17 May 2009; revised manuscript received 23 August 2009; published 21 September 2009)

\begin{abstract}
Attractive electron correlations due to an electron-vibron interaction can overcome the direct Coulomb repulsion of polarons in deformable molecular quantum dots (MQDs). If it happens, a switching appears in the $I-V$ characteristics of the degenerate nonadiabatic molecular bridges weakly coupled with electrodes providing a route to ultrafast "memristors" (memory resistors) as the basis for future oscillators, amplifiers and other important circuit elements. Here, we extend our theory of polaronic memristors to adiabatic MQDs strongly coupled with leads to show that the degeneracy of MQD (or multilevel energy structure) along with the polaron-polaron attraction is a vital ingredient of its switching behavior in the strong-coupling regime as well.
\end{abstract}

DOI: 10.1103/PhysRevB.80.115321

PACS number(s): 73.23.Hk, 63.22.Gh, 62.23.Hj, 71.38.Mx

\section{INTRODUCTION}

Different nanosize devices are being proposed and investigated ${ }^{1-3}$ that exhibit some kind of current "switching" behavior, 4,5 "negative differential resistance," and "memory." There is currently a surge of interest in various systems showing memristor behavior (see, for example, Ref. 7 and references therein) that can potentially be used for resistive random access memories. The practical significance of determining a precise microscopic mechanism of such a behavior is difficult to overestimate.

Polarons-electrons strongly coupled with lattice vibrations (phonons or vibrons) - play a key role in the transport and optical properties of many systems of reduced dimension and dimensionality. ${ }^{8-11}$ They may provide an almost instantaneous switching mechanism ( $\sim$ tens of $\mathrm{THz}){ }^{12}$ when a bistable current state of vibrating nanocircuits appears due to attractive electron-electron correlations ("anti-Coulomb blockade" of the insulating state ${ }^{13,14}$ ), if MQD is many-fold degenerate. Later on Galperin et al. ${ }^{15}$ argued, without discussing the discrepancies with the prior work, ${ }^{12}$ that even a nondegenerate electronic level coupled to a single vibrational mode produces a hysteretic $I-V$ curve, a current switching, and a negative differential resistance. We have explicitly calculated $I-V$ curves of the nondegenerate (or twofold degenerate) MQDs (Ref. 16) to show that these findings originate in an erroneous mean-field approximation (MFA) used in Ref. 15, which replaces the electron occupation number operator in electron-vibron interaction (EVI) by an average population of the molecular level. The correlations beyond this MFA are crucial to obtain correct results as explained in detail in Ref. 16.

The exact analytical solution of the problem fully accounting for the strong EVI and polaron-polaron correlations in MQD has been found by us at any temperature in the weak molecular-lead coupling limit, where the inverse lifetime, $\Gamma / \hbar$, of a polaron on the dot is small compared with the characteristic vibron frequency, $\Gamma / \hbar \ll \omega_{0}$ (Ref. 12) (nonadiabatic regime). Here, we extend our theory of polaronic memristors to MQDs strongly coupled with the leads, where $\Gamma$ $\gg \hbar \omega_{0}$ (adiabatic regime).

\section{MOLECULAR QUANTUM DOT: EXACT SOLUTION IN NONADIBATIC REGIME}

We have defined MQD by a molecular Hamiltonian, which includes the Coulomb repulsion, $U^{C}$, and the electronvibron interaction ${ }^{12}$

$$
\begin{aligned}
H_{m}= & \sum_{\mu} \varepsilon_{\mu} \hat{n}_{\mu}+\frac{1}{2} \sum_{\mu \neq \mu^{\prime}} U_{\mu \mu^{\prime}}^{C} \hat{n}_{\mu} \hat{n}_{\mu^{\prime}}+\sum_{\mu, q} \omega_{q} \hat{n}_{\mu}\left(\gamma_{\mu q} d_{q}+\text { H.c. }\right) \\
& +\sum_{q} \omega_{q}\left(d_{q}^{\dagger} d_{q}+1 / 2\right) .
\end{aligned}
$$

Here $\varepsilon_{\mu}$ are the energy levels of a rigid molecule, $\hat{n}_{\mu}=c_{\mu}^{\dagger} c_{\mu}$ is the molecular occupation number operator, $d_{q}$ annihilates vibron, $\omega_{q}$ is the vibron frequency, and $\gamma_{\mu q}$ is the EVI constant ( $q$ enumerates the vibron modes and we take $\hbar=k_{B}=1$ here and below). This Hamiltonian conserves the occupation numbers of molecular states $n_{\mu}$. When the molecule is attached to two (for simplicity symmetric) leads, the full Hamiltonian $H=H_{m}+H_{l}+\Sigma_{\mu, k} t_{k \mu} c_{\mu}^{\dagger}\left(a_{k}+b_{k}\right)+$ H.c. includes the hopping to the leads, and the Hamiltonian of the leads $H_{l}=\Sigma_{k} \xi_{k}\left(a_{k}^{\dagger} a_{k}+b_{k}^{\dagger} b_{k}\right)$, where $a_{k}$ and $b_{k}$ annihilate the electron in the left and right electrodes, respectively.

With some conventional assumptions, such as energyindependent width $\Gamma=2 \pi \Sigma_{k} t_{k \mu}^{2} \delta\left(E-\xi_{k}\right)$ and quasiequilibrium electron distributions in the leads, the current through MQD is given by the Landauer-type expression (or by the Fermi golden rule) as ${ }^{12}$

$$
I(V)=I_{0} \int_{-\infty}^{\infty} d E\left[f_{1}(E)-f_{2}(E)\right] \rho(E),
$$

allowing for a transparent analysis of the essential physics of the switching phenomenon. Here, $I_{0}=e \Gamma, f_{1(2)}(E)$ $=1 /\{\exp [\beta(E+\Delta \mp e V / 2)]+1\}$ is the electron distribution function in (1) left and (2) right metallic leads, respectively, $\beta=1 / T$ is the inverse temperature, $\Delta$ is the position of the lowest unoccupied molecular level with respect to the Fermi level at $V=0$, and $V$ is the voltage drop across the MQD.

The molecular density of states (DOS) $\rho(E)$ $=-(1 / \pi) \Sigma_{\mu} \operatorname{Im} G_{\mu}^{R}(E)$ generally depends on all interactions and molecule-lead hopping integrals as determined by the 
Fourier component $G_{\mu}^{R}(E)$ of the retarded Green's function (GF) $G_{\mu}^{R}(t)=-i \Theta(t)\left\langle c_{\mu}(t) c_{\mu}^{\dagger}+c_{\mu}^{\dagger} c_{\mu}(t)\right\rangle$, where $c_{\mu}(t)$ is the Heisenberg annihilation operator. To calculate $\rho(E)$, one can neglect molecule-lead coupling in the weak-coupling regime while keeping all orders of the Coulomb repulsion and EVI by means of the canonical displacement transformation of the molecular Hamiltonian (1). In particular, assuming EVI with a single vibronic mode, $\omega_{q}=\omega_{0}$ and the coupling $\gamma_{\mu q}$ $=\gamma$, and constant Coulomb integrals, $U_{\mu \mu^{\prime}}^{C}=V_{c}$, one obtains for a $d$-fold degenerate single-level $\operatorname{dot}^{12^{\prime}}$

$$
\begin{aligned}
\rho(E)= & \mathcal{Z} d \sum_{r=0}^{d-1} Z_{r}(n) \sum_{l=0}^{\infty} I_{l}(\xi) \\
& \times\left\{e^{\beta \omega_{0} l / 2}\left[(1-n) \delta\left(E-r U-l \omega_{0}\right)+n \delta\left(E-r U+l \omega_{0}\right)\right]\right. \\
& +\left(1-\delta_{l 0}\right) e^{-\beta \omega_{0} l / 2}\left[n \delta\left(E-r U-l \omega_{0}\right)\right. \\
& \left.\left.+(1-n) \delta\left(E-r U+l \omega_{0}\right)\right]\right\} .
\end{aligned}
$$

Here $\mathcal{Z}=\exp \left[-|\gamma|^{2} \operatorname{coth}\left(\beta \hbar \omega_{0} / 2\right)\right]$ accounts for a familiar polaronic renormalization of the hopping integrals, $\xi$ $=|\gamma|^{2} / \sinh \left(\beta \hbar \omega_{0} / 2\right), I_{l}(\xi)$ is the modified Bessel function, and $\delta_{l k}$ is the Kroneker symbol $(l, k=0,1,2, \ldots)$, and we take the position of the level as zero, $\epsilon_{\mu}=0$. The resulting polaron-polaron interaction, $U=V_{c}-2 E_{p}$, where $E_{p}=|\gamma|^{2} \omega_{0}$ is the polaron level shift, comprises the Coulomb repulsion, $V_{c}$, and the vibron-mediated attraction. An important feature of DOS [Eq. (3)] is its nonlinear dependence on the occupation number $n$ of the degenerate molecular states owing to the correlation side bands with the spectral weight $Z_{r}(n)=(d$ $-1) ! n^{r}(1-n)^{d-1-r} /[r !(d-1-r) !]$. The DOS, Eq. (3), contains full information about all possible correlations in transport, in particular, the vibron and correlation side bands. It is derived by solving the finite system of coupled equations for $\mathrm{N}$-particle Green's functions, as described in Ref. 12.

Equating incoming and outgoing numbers of electrons in MQD per unit time, one obtains the self-consistent equation for the molecular-state occupation number $n$ as ${ }^{12}$

$$
2 n d=\int d E \rho(E)\left[f_{1}(E)+f_{2}(E)\right]
$$

which automatically satisfies $0 \leq n \leq 1$. In particular, for the nondegenerate MQD $(d=1)$ and $T=0 \mathrm{~K}$ the result is

$$
n=\frac{b_{0}^{+}}{2+b_{0}^{+}-a_{0}^{+}}
$$

and

$$
\frac{I}{I_{0}}=\frac{2 b_{0}^{-}+a_{0}^{-} b_{0}^{+}-a_{0}^{+} b_{0}^{-}}{2+b_{0}^{+}-a_{0}^{+}} .
$$

where $\quad a_{0}^{ \pm}=\mathcal{Z} \Sigma_{l=0}^{\infty}\left(|\gamma|^{2 l} / l !\right)\left[\Theta\left(l \omega_{0}-\Delta+e V / 2\right) \pm \Theta\left(l \omega_{0}-\Delta\right.\right.$ $-e V / 2)]$ and $b_{0}^{ \pm}=\mathcal{Z} \Sigma_{l=0}^{\infty}\left(|\gamma|^{2 l} / l !\right)\left[\Theta\left(-l \omega_{0}-\Delta+e V / 2\right) \pm \Theta(\right.$ $\left.\left.-l \omega_{0}-\Delta-e V / 2\right)\right]$. Here $\Theta(x)=1$ if $x>0$ and zero otherwise. The current is single valued, Fig. 1, with the familiar ladder due to the phonon side bands. ${ }^{17}$ The double-degenerate level $d=2$ provides more elementary processes for conductance reflected in a larger number of steps on the phonon ladder in comparison to $d=1$ case. However, the current remains also

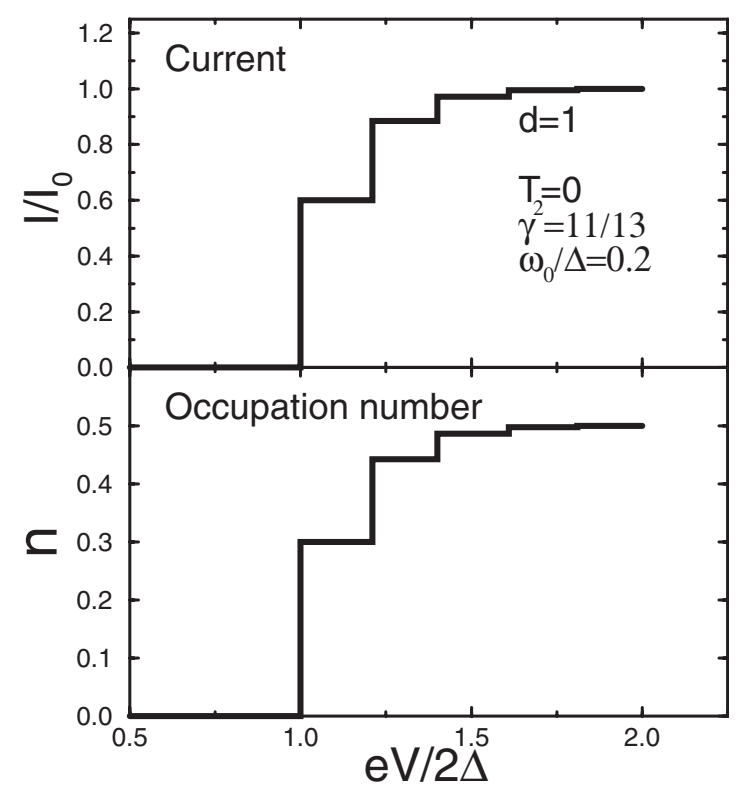

FIG. 1. Current-voltage characteristic of the nondegenerate $(d$ =1) MQD weakly coupled to the leads at $T=0 \mathrm{~K}, \omega_{0} / \Delta=0.2$, and $\gamma^{2}=11 / 13$. There is the vibron ladder in $I-V$ but no hysteresis.

single valued showing no switching behavior ${ }^{11,12}$ in the weak molecular-lead coupling regime, contrary to the MFA of Ref. 15 , which yields an artificial switching behavior for a nondegenerate level, $d=1$, in this regime. The switching appears only in $I$ - $V \mathrm{~s}$ of many-fold degenerate dot when $U<0$ and the temperature is low enough. ${ }^{2,13}$

\section{ADIABATIC MQD: NONEQUILIBRIUM LINKED CLUSTER EXPANSION}

Let us now analyze an effect of the strong molecular-lead coupling $\Gamma \gg \omega_{0}$ on the above results. When EVI is also large $(\gamma \gg 1)$, it could not be treated perturbatively, so that one has to sum up all diagrams of the perturbation expansion in powers of $\gamma$ to calculate GFs. As proposed in Ref. 18, this can be done within a lowest-order nonequilibrium linked cluster expansion (NLCE),${ }^{19}$ which provides approximate resummation of the whole series for GFs as

$$
G(t) \equiv \sum_{r=0}^{\infty} \gamma^{2 r} W_{r}(t) \approx G_{0}(t) \exp \left[\gamma^{2} F(t)\right] .
$$

Here, $G_{0}(t)$ should be calculated without EVI [i.e., with $\gamma_{\mu q}=0$ in Eq. (1)] while $F(t)$ is found using the lowest (second)-order diagrams $F(t)=G_{0}^{-1}(t) W_{1}(t)$. Calculating zero $G_{0}(t)$ and second order (in $\left.\gamma\right) W_{1}(t)$ is a textbook exercise for the nondegenerate molecular level. Using equations of motion, $i \dot{c}=t \sum_{k}\left(a_{k}+b_{k}\right)$ and $i \dot{a}_{k}=\xi_{k} a_{k}+t c, i \dot{b}_{k}=\xi_{k} b_{k}+t c$ one can readily find the Fourier component of the retarded GF of the rigid nondegenerate dot as $G_{0}^{R}(E)=(E-i \Gamma)^{-1}$ and a Lorentzian-type zero-order molecular DOS

$$
\rho_{0}(E)=\frac{1}{\pi} \frac{\Gamma}{E^{2}+\Gamma^{2}} .
$$

Substituting Eq. (8) into Eq. (2) yields the current through a 

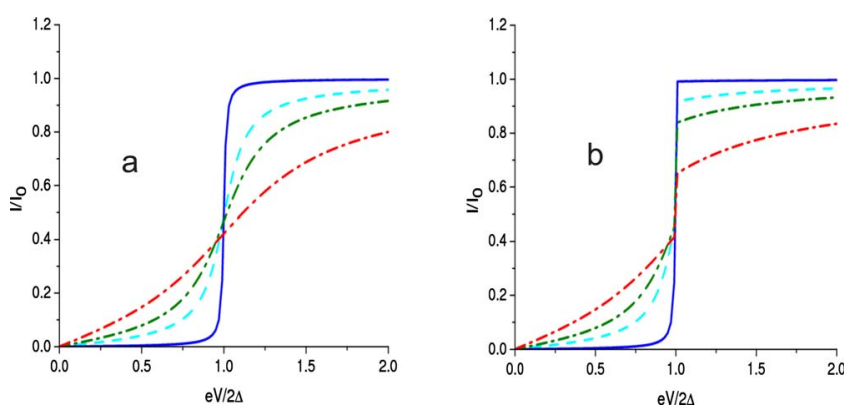

FIG. 2. (Color online) Current-voltage characteristics of the nondegenerate $(d=1)$ rigid bridge (a) $T=0 \mathrm{~K}$, and (b) of the nondegenerate adiabatic $\left(\omega_{0} \ll \Gamma\right)$ molecular bridge with the polaron level shift $E_{p}=\Delta / 2$ for different couplings to the leads, $\Gamma / \Delta=0.01$ (the steepest curve), 0.1,0.2, and 0.5 .

rigid bridge as

$$
\frac{I^{(0)}}{I_{0}}=\frac{1}{\pi}\left[\arctan \left(\frac{e V / 2-\Delta}{\Gamma}\right)+\arctan \left(\frac{e V / 2+\Delta}{\Gamma}\right)\right],
$$

shown in Fig. 2(a) at $T=0 \mathrm{~K}$ for a few inverse lifetimes.

Using the zero-order lesser, $G_{0}^{<}(t) \approx i n_{0} \exp (-\Gamma|t|)$, and greater, $G_{0}^{>}(t) \approx-i\left(1-n_{0}\right) \exp (-\Gamma|t|)$, GFs (Ref. 18) one can readily calculate the second-order contribution $W_{1}(t)$. Here $n_{0}=\left[f_{1}(0)+f_{2}(0)\right] / 2$ is the zero-order population of the molecular level. Then applying NLCE, Eq. (7), one gets the retarded GF for the strong molecular-lead coupling, $\Gamma \gg \omega_{0}$, summing infinite number of EVI diagrams (see for details Refs. 18 and 19), $G^{R}(E)=\left(E+2 n_{0} E_{p}-i \Gamma\right)^{-1}$, and the molecular DOS

$$
\rho(E)=\frac{1}{\pi} \frac{\Gamma}{\left(E+2 n_{0} E_{p}\right)^{2}+\Gamma^{2}} .
$$

Using Eq. (10) in Eqs. (4) and (2) leads to

$$
n=\frac{1}{2 \pi}\left[\pi+\arctan \left(\frac{e V / 2-\tilde{\Delta}}{\Gamma}\right)-\arctan \left(\frac{e V / 2+\tilde{\Delta}}{\Gamma}\right)\right]
$$

for the molecular-state population and

$$
\frac{I}{I_{0}}=\frac{1}{\pi}\left[\arctan \left(\frac{e V / 2-\tilde{\Delta}}{\Gamma}\right)+\arctan \left(\frac{e V / 2+\tilde{\Delta}}{\Gamma}\right)\right]
$$

for the current, respectively, with $\widetilde{\Delta} \equiv \Delta-2 n_{0} E_{p}=\Delta$ $-E_{p} \Theta(e V / 2-\Delta)$ at low temperatures, $T \ll \Gamma$. The level population, Eq. (11), of the nondegenerate dot strongly coupled to the leads, and the current, Eq. (12), Fig. 2(b) remain singlevalued showing neither switching nor negative differential resistance, similar to the weak molecular-lead coupling, Fig. 1. Taking into account the coupling with the leads beyond the second order washes out the vibron ladder in the $I-V$ characteristics, so that EVI affects the $I-V \mathrm{~s}$ only marginally compared with the rigid dot, Fig. 2, in the strong-coupling (adiabatic) regime.

As we have shown earlier ${ }^{16}$ the MFA of Ref. 15 replacing the occupation number operator $\hat{n}$ in EVI by the average population $n$ erroneously leads to a nonlinear equation for $n$ and a spurious switching of the nondegenerate dot, which has no physical meaning. The authors of Ref. 18 have confirmed our conclusion for the weak molecular-lead coupling but argued using NLCE that their MFA works well in the strong-coupling regime so that the nondegenerate adiabatic dot is multistable contrary to our present result, Fig. 2(b). The discrepancy originates in an erroneous replacement of the zero-order occupation $n_{0}$ in $G_{0}^{<,>}(t), G^{R}(E)$, and as the result in Eq. (10) by the exact $n$ leading to double counting of EVI diagrams in Ref. 18. In fact, multiple solutions for the steady-state population of the nondegenerate dot found in Refs. 15 and 18 are artifacts of MFA and misapplication of NLCE, respectively. Other studies ${ }^{20}$ find no hysteresis in non(or twofold-)degenerate dots either.

\section{SWITCHING OF ADIABATIC QUANTUM DOT}

The physical mechanism of the switching is provided by an attraction of two polarons in a multiply degenerate MQD, ${ }^{12-14}$ which is missing in the nondegenerate "polaron model" of Refs. 15 and 18 due to the Pauli exclusion principle.

Treating the polaron-polaron correlations in real multilevel bridges and the molecular-lead coupling at the same level of approximation is a challenging problem, which might require numerical techniques. ${ }^{21,22}$ Since the vibron ladders in $I-V$ curves are washed out by the strong coupling with the leads, Fig. 2(b), we consider here an effect of the molecular-lead coupling on the switching in the many-fold degenerate $(d \gg 1)$ negative Hubbard $U$ model of Ref. 13 allowing for a simple analytical solution

$$
\begin{aligned}
H= & \frac{1}{2} U \sum_{\mu \neq \mu^{\prime}} \hat{n}_{\mu} \hat{n}_{\mu^{\prime}}+\sum_{k} \xi_{k}\left(a_{k}^{\dagger} a_{k}+b_{k}^{\dagger} b_{k}\right) \\
& +\sum_{k, \mu}\left[t_{k \mu}\left(a_{k}^{\dagger}+b_{k}^{\dagger}\right) c_{\mu}+\text { H.c. }\right] .
\end{aligned}
$$

For the many-fold degenerate dot, one can approximate the exact two-body interaction in the Hamiltonian Eq. (13) by a single-particle self-consistent Hartree-type potential as $\frac{1}{2} U \Sigma_{\mu \neq \mu^{\prime}} \hat{n}_{\mu} \hat{n}_{\mu^{\prime}} \approx U \Sigma_{\mu \neq \mu^{\prime}} \hat{n}_{\mu} n_{\mu^{\prime}}-\frac{1}{2} U \Sigma_{\mu \neq \mu^{\prime}} n_{\mu} n_{\mu^{\prime}}$. Then, using equations of motions yields the DOS as in the nondegenerate rigid dot, Eq. (8), but shifted in energy by the attraction potential

$$
\rho(E)=\frac{1}{\pi} \frac{d \Gamma}{[E-U(d-1) n]^{2}+\Gamma^{2}} .
$$

From Eq. (4), the molecular-state population for $T \ll \Gamma$ is

$$
\begin{aligned}
n= & \frac{1}{2 \pi}\left[\pi+\arctan \left(\frac{e V / 2-\Delta-2 \tilde{U} n}{\Gamma}\right)\right. \\
& \left.-\arctan \left(\frac{e V / 2+\Delta+2 \tilde{U} n}{\Gamma}\right)\right],
\end{aligned}
$$

where $\tilde{U}=U(d-1) / 2$.

For $\Gamma=0$, there are two stable solutions of Eq. (15), $n$ $=0$ and $n=0.5$ in the voltage region of bistability, $\Delta-|\tilde{U}|$ 


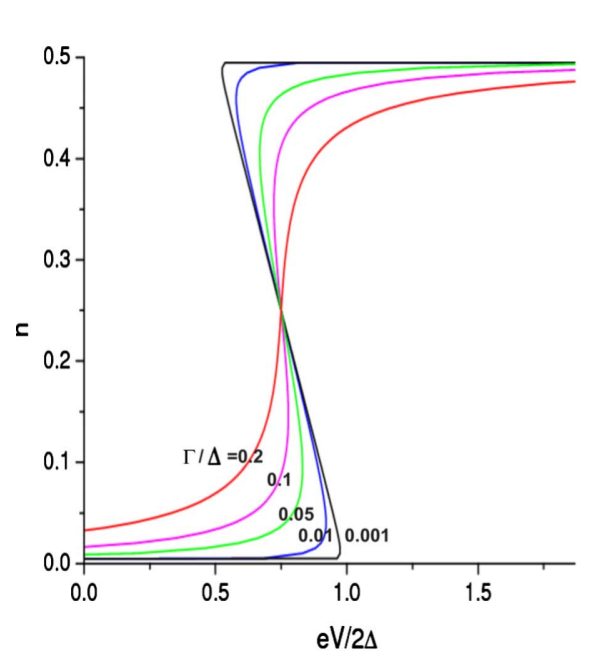

$<e V / 2<\Delta$, and only one solution, $n=0.5$ for $e V / 2>\Delta$, and one solution $n=0$ for $e V / 2<\Delta-|\tilde{U}|$. The coupling with the leads does not destroy the many-fold degenerate memristor as long as $\Gamma$ is less than some critical value, $\Gamma<\Gamma_{c}$, which is small compared with $\Delta$, Fig. 3 . When $\Gamma \ll \Delta$, one can replace the last arctan in Eq. (15) by $\pi / 2$ and resolve this transcendental equation with respect to the bias voltage as $e V / 2$ $\approx \Delta+2 \tilde{U} n-\Gamma \cot (2 \pi n)$. The boundary of bistability is found from $d V / d n=0$, which yields the terminal value of the level width $\Gamma_{c}=|\tilde{U}| / \pi$. The dot population and the current, found from Eq. (2) using Eqs. (14) and (15) remain double valued in the voltage region of bistability, Fig. 3, where both highcurrent and low-current branches are stable, while the intermediate branch with $d n / d V<0$, Fig. 3(a), is unstable. ${ }^{13}$ Importantly, the coupling narrows the voltage range of the hysteresis loop but the transition from the low-(high)-current branch to the high-(low)-current branch remains discontinuous as long as $\Gamma<\Gamma_{c}$. The Hartree-type approximation for the correlation potential is fully justified by the exact solution of Ref. 13 in the nonadibatic regime. More complicated electron and vibron-energy spectra of MQD should have no qualitative effect on the switching as soon as the characteristic attraction $|\tilde{U}|$ remains larger than the widths of both spectra. $^{13}$

Different from the nondegenerate and twofold-degenerate dots, the rate equation for a multidegenerate dot, $d>2$, has multiple physical roots in a certain voltage range showing hysteretic behavior due to attractive correlations between different electronic states of MQD. The origin of the bistability is illustrated in Fig. 4. When the current flows through MQD, the "highest occupied molecular orbital-lowest unoccupied molecular orbital" (HOMO-LUMO) gap is renormalized down to a lower value due to attractive correlations between populated molecular levels, so the current-off voltage $V_{1}$ turns out smaller than the current-on voltage $V_{2}$.

\section{CONCLUSION}

The actual mechanisms of nanocircuit switching are of the highest experimental and theoretical value. Further progress will depend upon understanding of intrinsic mechanisms of nanomemristors. Here, we have extended our multipolaron theory of the current controlled switching mechanism, Fig. 4 to the adiabatic molecular-size bridges with the significant coupling to the leads $\left(\Gamma \gg \omega_{0}\right)$. The degenerate (or multilevel) MQD shows the hysteretic memory, if the degeneracy of the level (or the number of levels) is large enough, the Coulomb repulsion is overscreened by EVI (Ref. 23) or by any other attractive mechanism, and the coupling to the leads is below the critical value, Fig. 3. Recent unbiased exact diagonalization studies of the very similar Holstein-Hubbard model $^{22}$ show indeed that EVI can overcome the direct Coulomb repulsion in MQD and the multilevel energy structure of the deformable quantum dot is vital for the switching. The time scale for the switching is the inverse vibron frequency $(\sim$ tens of THz). Importantly, the switching does not exist in nondegenerate molecular dots neither weakly, Fig. 1, nor strongly coupled to the leads, Fig. 2(b), contrary to the claims in Refs. 3, 15, and 18. In many-fold degenerate MQDs, bistability is destroyed by a very strong coupling to

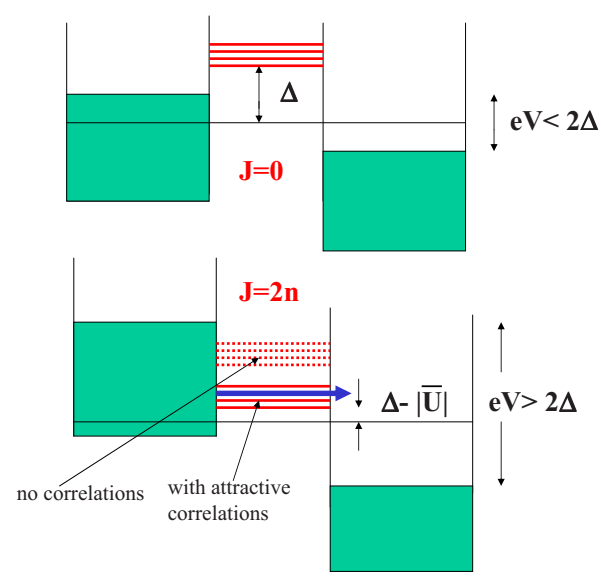

FIG. 4. (Color online) Schematic of energy levels of the molecular quantum dot under bias voltage $V$. MQD is assumed to be fourfold degenerate $(d=4)$. Switching occurs in the voltage range $V_{1}<V<V_{2}\left(e V_{1}=2(\Delta-|\widetilde{U}|)\right.$ and $\left.e V_{2}=2 \Delta\right)$ due to a lowering of the HOMO-LUMO gap by the attractive electron-electron potential $U$ in the current state $J \neq 0$ (after Ref. 10). 
the leads, $\Gamma>\Gamma_{c}$ and/or at temperatures above some critical value. $^{12}$

\section{ACKNOWLEDGMENT}

This work was supported by EPSRC (U.K.) (Grant No.
EP/H004483). We greatly appreciate enlightening discussions with Stanley Williams, in particular his comment that our polaronic memristor is generally different from the more conventional memory resistors, which are defined as the ones exhibiting bipolar nonvolatile switching.
${ }^{1}$ Molecular Electronics: Science and Technology, edited by A. Aviram and M. Ratner (Ann. N.Y. Acad. Sci, New York, 1998).

${ }^{2}$ C. P. Collier, E. W. Wong, M. Belohradsky, F. M. Raymo, J. F. Stoddart, P. J. Kuekes, R. S. Williams, and J. R. Heath, Science 285, 391 (1999); D. I. Gittins, D. Bethell, D. J. Schiffrin, and R. J. Nichols, Nature (London) 408, 67 (2000).

${ }^{3}$ M. Galperin, M. A. Ratner, and A. Nitzan, J. Phys.: Condens. Matter 19, 103201 (2007).

${ }^{4}$ Z. J. Donhauser, B. A. Mantooth, K. F. Kelly, L. A. Bumm, J. D. Monnell, J. J. Stapleton, D. W. Price, Jr., A. M. Rawlett, D. L. Allara, J. M. Tour, and P. S. Weiss, Science 292, 2303 (2001).

${ }^{5}$ D. R. Stewart, D. A. A. Ohlberg, P. A. Beck, Y. Chen, R. S. Williams, J. O. Jeppesen, K. A. Nielsen, and J. F. Stoddart, Nano Lett. 4, 133 (2004).

${ }^{6}$ R. Waser and M. Aono, Nature Mater. 6, 833 (2007).

${ }^{7}$ D. B. Strukov, G. S. Snider, D. R. Stewart, and R. S. Williams, Nature (London) 453, 80 (2008); J. J. Yang, M. D. Pickett, X. M. Li, Douglas A. A. Ohlberg, Duncan R. Stewart, and R. Stanley Williams, Nat. Nanotechnol. 3, 429 (2008); D. Ielmini, C. Cagli, and F. Nardi, Appl. Phys. Lett. 94, 063511 (2009).

${ }^{8}$ J. T. Devreese, in Handbook of Semiconductor Nanostructures and Nanodevices, edited by A. A. Balandin and K. L. Wang (American Scientific, Los Angeles, 2006) 4, 311.

${ }^{9}$ S. N. Klimin, V. M. Fomin, F. Brosens, and J. T. Devreese, Phys. Rev. B 69, 235324 (2004).

${ }^{10}$ A. S. Alexandrov, in Nanotechnology for Electronic Materials and Devices Series: Nanostructure Science and Technology, ed- ited by A. Korkin, E. Gusev, J. K. Labanowski, and S. Luryi (Springer, New York, 2006), p. 306.

${ }^{11}$ A. M. Bratkovsky, in Polarons in Advanced Materials, edited by A. S. Alexandrov (Canopus/Springer, Bristol, 2007), p. 623.

${ }^{12}$ A. S. Alexandrov and A. M. Bratkovsky, Phys. Rev. B 67, 235312 (2003).

${ }^{13}$ A. S. Alexandrov, A. M. Bratkovsky, and R. S. Williams, Phys. Rev. B 67, 075301 (2003).

${ }^{14}$ V. N. Ermakov, Physica E 8, 99 (2000).

${ }^{15}$ M. Galperin, M. A. Ratner, and A. Nitzan, Nano Lett. 5, 125 (2005).

${ }^{16}$ A. S. Alexandrov and A. M. Bratkovsky, J. Phys.: Condens. Matter 19, 255203 (2007).

${ }^{17}$ S. Braig and K. Flensberg, Phys. Rev. B 68, 205324 (2003); K. Flensberg, ibid. 68, 205323 (2003).

${ }^{18}$ M. Galperin, A. Nitzan, and M. A. Ratner, J. Phys.: Condens. Matter 20, 374107 (2008).

${ }^{19}$ P. Kral, Phys. Rev. B 56, 7293 (1997).

${ }^{20}$ A. Mitra, I. Aleiner, and A. J. Millis, Phys. Rev. B 69, 245302 (2004); Phys. Rev. Lett. 94, 076404 (2005); P. S. Cornaglia, H. Ness, and D. R. Grempel, ibid. 93, 147201 (2004).

${ }^{21}$ R. Zitko and J. Bonca, Phys. Rev. B 74, 224411 (2006).

${ }^{22}$ H. Fehske, G. Wellein, J. Loos, and A. R. Bishop, Phys. Rev. B 77, 085117 (2008).

${ }^{23}$ See, e.g., E. G. Maksimov and O. V. Dolgov, Phys. Usp. 50, 933 (2007) for a general discussion of the overscreening effects. 